\title{
Comparison of hepatic artery reconstruction using surgical loupe and operating microscope during living donor liver transplantation focusing on the beginner's point
}

\author{
Eun Kyoung Jwa, Joo Dong Kim, and Dong Lak Choi \\ Division of Hepatobiliary Pancreas Surgery and Abdominal Organ Transplantation, Department of Surgery, \\ Catholic University of Daegu College of Medicine, Daegu, Korea
}

\begin{abstract}
Backgrounds/Aims: Hepatic artery (HA) reconstruction during living donor liver transplantation (LDLT) has been performed by experienced microsurgeons with operative microscope in most centers. However, it takes long time to learn the skills and so, to simplify this procedure, transplant surgeons recently performed this procedure using surgical loupe. Methods: This study retrospectively reviewed outcomes of 237 LDLTs at our institution from January 2012 to October 2016. In group I, HA reconstruction was performed under operative microscope by an experienced microsurgeon and in group II, it was performed using surgical loupe by a transplant surgeon with little experience for arterial anastomosis. Results: There was no difference in most perioperative outcomes between two groups except mean time required for HA reconstruction $(24.2 \pm 4.3$ vs. $20.9 \pm 6.9$ minutes, $p=0.001)$. Multivariable regression modeling to adjust for baseline differences showed that the use of surgical loupe was not associated with either HA thrombosis or intraoperative HA revision rate. Conclusions: HA reconstruction under surgical loupe can be performed simply and yields results as good as with operative microscopy, even when the transplant surgeon has less experience with HA anastomosis. (Ann Hepatobiliary Pancreat Surg 2019;23:122-127)
\end{abstract}

Key Words: Hepatic artery; Microscope; Loupe; Living donor; Liver transplantation

\section{INTRODUCTION}

Living donor liver transplantation (LDLT) has gradually been accepted as the best treatment option for patents with end-stage liver disease because of the shortage of deceased donors. ${ }^{1,2}$ However, the arterial reconstruction during LDLT is technically more difficult than in deceased donor liver transplantation (DDLT) because of the small diameter of the vessels in partial liver grafts. ${ }^{3-5}$ The use of an operating microscope was adopted initially because of the high hepatic artery thrombosis (HAT) rates and the use of microscopic surgery during LDLT significantly reduced the incidence of HAT and improved graft survival. ${ }^{4,6-8}$ Therefore, in most centers, an experienced microsurgeon establishes hepatic artery (HA) flow using an operating microscope. ${ }^{3,9}$ However, it takes a long time to learn the skills involved in microsurgical reconstruction and to gain sufficient experience to achieve good outcomes. ${ }^{3,9,10}$ In addition, the HA reconstruction takes longer because of the deep operative field, shorter HA graft, and size discrepancy between the donor and recipient HA. ${ }^{3}$ With increasing experience in LDLT, some surgeons have reported that loupe magnification can achieve similar results in LDLT without the need for a microscope. ${ }^{3,9}$ Therefore, this study retrospectively reviewed our experience with HA reconstruction in adult-to-adult LDLT and describes the excellent outcomes without an operating microscope especially focusing on the beginner's point.

Received: June 29, 2018; Revised: November 5, 2018; Accepted: November 15, 2018

Corresponding author: Joo Dong Kim

Division of Hepatobiliary Pancreas Surgery and Abdominal Organ Transplantation, Department of Surgery, Catholic University of Daegu College of Medicine, 33 Duryugongwon-ro 17-gil, Nam-gu, Daegu 42472, Korea

Tel: +82-53-650-3074, Fax: +82-53-650-4950, E-mail: milledr@cu.ac.kr

Copyright (C) 2019 by The Korean Association of Hepato-Biliary-Pancreatic Surgery

This is an Open Access article distributed under the terms of the Creative Commons Attribution Non-Commercial License (http://creativecommons.org/ censes/by-nc/4.0) which permits unrestricted non-commercial use, distribution, and reproduction in any medium, provided the original work is properly cited. Annals of Hepato-Biliary-Pancreatic Surgery • pISSN: 2508-5778 - eISSN: 2508-5859 


\section{PATIENTS AND METHODS}

\section{Patients}

Between January 2012 and October 2016, we retrospectively studied the records of 237 LDLT patients using data collected among 241 cases of consecutive adult LDLT performed at our institution. The medical records were reviewed retrospectively in terms of patient demographics and intra- and postoperative findings, including patient age, disease etiology, laboratory data, operative outcomes, postoperative complications, and long-term outcomes. For standardization purposes, 4 patients in the period primarily using a surgical loupe were excluded due to only availability of operative microscope for the following cases: right posterior sector grafts with too small caliber and short stump of donor HA or right lobe grafts with double donor HA stumps which have both a smaller caliber and short length. The study was approved by the Institutional Review Board of our institution. The patients were divided into two groups, depending on whether an operative microscope or surgical loupe was used during HA reconstruction. In group I ( $\mathrm{n}=136)$, HA reconstruction was performed under a surgical microscope by an experienced microsurgeon until September 2014. Subsequently, a transplant surgeon who had no experience in arterial anastomosis during LDLT performed the HA reconstruction using a surgical $5 \times$ loupe (group II, $\mathrm{n}=101$ ). He only had experience in vascular anastomosis in large vessels such as the hepatic vein or portal vein during LDLT and received no prior microsurgical training. The mean follow-up period was $50.9 \pm 17.1$ months in group I and 23.5 \pm 9.1 months in group II $(p=0.000)$. However, this difference was due to the historical nature of the two groups.

\section{Surgical techniques and follow-up for graft patency}

The detailed surgical procedure used for HA reconstruction is described elsewhere and the anastomosis procedures were similar in the two groups. ${ }^{10,11}$ The recipient right and left hepatic arteries can be dissected out higher in the hilum to maximize artery length and mobility for the application of double microvascular clamps. After adequate debridement, the quality of the recipient HA was inspected carefully, including the interior of the vessel and the strength of the pulsatile arterial flow. A double micro- clamp is first applied to the donor HA and then to the recipient HA after aligning both terms of length and rotation. An end-to-end anastomosis is performed using interrupted 8-0 Nylon sutures with the aid of an operating microscope or surgical loupe. We didn't perform continuous suturing in all cases. First, both the dorsal and ventral ends were anastomosed and four or five sutures were placed while first two staying sutures were stretched apart gently by first assistant. Back wall stiches were placed in similar fashion after the micro-clamp were rotated $180^{\circ}$ keeping two corner sutures stretched like conventional twist technique. The back wall-first anastomosis technique was employed in cases which couldn't flip the artery due to inadequate vessel length.

Immediately after completion of the vascular reconstruction, intraoperative Doppler ultrasound (DUS) is performed routinely to check adequacy of the HA and portal vein inflow and they were addressed immediately if the intraoperative DUS findings were abnormal. If there was no HA flow during intraoperative assessment, after a brief observation, we tried to do HA anastomosis repeatedly to establish the arterial flow. If the cause of low HA flow is arterial spasm, we advocate a brief waiting period for the spasm to relieve and to rule out any anatomical factors such as kinking, angulation or thrombosis. However, if this is not related to HA spasm, we performed immediate revision of the HA anastomosis.

DUS was performed on post-transplant days 1 and 3, and dynamic computed tomography (CT) scans were performed on days 7 and 14 to assess the inflow and outflow graft patency in the early posttransplant period. If hepatic arterial or portal vein complications were suspected during DUS with elevated liver enzymes, those complications were further confirmed by a CT angiography.

\section{Postoperative management}

The immunosuppression regimen consisted of calcineurin inhibitors, mycophenolate mofetil and low-dose steroids. Fresh-frozen plasma was transfused only when massive bleeding and oozing were observed. Prostaglandin E1 was administrated intravenously immediately following graft reperfusion and continued for seven days. All patients were not heparinized in the postoperative period as prophylaxis. Acetylsalicylic acid $100 \mathrm{mg}$ /day was started when the patient tolerated oral intake. 


\section{Statistical analysis}

All numerical data are reported as the mean and standard deviation. Student's $t$-test or the Mann-Whitney $U$ test was used to compare continuous variables according to their distributions. The chi-square test or Fisher's exact test was used to compare categorical variables after assumptions were verified. Survival and patency rates were determined with the Kaplan-Meier method and were compared with the log-rank test. Multivariate analysis was applied to assess the statistical association and risk factors for HAT and intraoperative HA revision. Multivariate analyses with logistic regression were performed to quantify the adjusted odds ratio (OR) to control for established risk factors and to assess the independent impact of the use of surgical loupe on postoperative outcome. All analyses were performed using SPSS Statistics ver. 19.0 (IBM, Armonk, NY, USA). A value of $p<0.05$ was taken to indicate statistical significance.

\section{RESULTS}

\section{Patient and perioperative characteristics}

Table 1 summarizes the recipient and donor demographic data. No significant difference was observed between the two groups, except for recipient age and incidence of ABO-incompatible LDLT. The difference in the incidence of ABO-incompatible LDLT was only due to the chronological nature of the two groups. The principal etiology of liver disease was viral hepatitis-related liv- er cirrhosis in 153 patients (101 of whom also had hepatocellular carcinoma). The mean recipient and donor ages were $51.7 \pm 9.1$ and $31.5 \pm 11.4$ years old, respectively. The mean score for model for end-stage liver disease (MELD) was $17.0 \pm 10.1$. Thirty-seven recipients (15.6\%) underwent ABO-incompatible LDLT. A single HA anastomosis was performed in 223 patients (94.1\%), while double anastomoses were performed in 14 cases $(5.9 \%)$ and the mean donor HA diameter also did not differ between the two groups. The incidence of intraoperative HA revision was not different between the two groups, and the most common cause was thrombosis due to a poor HA condition and intimal dissection. The HA reconstruction took longer in group I than in group II, although the HA reconstruction was performed by a more experienced microsurgeon in group I $(p=0.001)$. No other perioperative variables differed significantly between the two groups (Table 2).

In multivariate logistic regression analyses, only significant hilar fibrosis (OR, 22.033; 95\% confidence interval [CI], 1.179-411.899; $p=0.038$ ) and a smaller HA diameter (defined as less than $1.8 \mathrm{~mm}$ ) (OR, 0.002; 95\% CI, 0.000$0.166 ; p=0.007)$ were associated with HA thrombosis (HAT) but not related to the use of surgical loupe (OR, 0.969; 95\% CI, 0.0076-12.351; $p=0.981$ ) when adjusted for the established risk factors of HAT such as recipient age, sex, previous hepatectomy, pretransplant locoregional therapy including transarterial chemoembolization, portal vein thrombosis or stenosis, use of surgical loupe, significant hilar fibrosis which cause difficult dissection, multi-

Table 1. Demographic characteristics of the donors and recipients

\begin{tabular}{lccc}
\hline \multicolumn{1}{c}{ Variable } & MS group (n=136) & SL group (n=101) & $p$-value \\
\hline Recipient age (year) & $50.2 \pm 9.5$ & $53.7 \pm 8.1$ & 0.003 \\
Recipient sex, male/female & $90 / 46$ & $73 / 28$ & 0.316 \\
Original disease (n, \%) & & & 0.188 \\
$\quad$ Viral hepatitis & $83(61.0)$ & $70(69.3)$ & \\
$\quad$ Others & $33(39.0)$ & $31(30.7)$ & 0.293 \\
Accompanying HCC (n, \%) & $54(39.7)$ & $47(46.5)$ & 0.502 \\
CTP score & $8.2 \pm 2.5$ & $7.9 \pm 2.6$ & 0.329 \\
MELD score & $17.6 \pm 10.8$ & $16.3 \pm 8.9$ & 0.238 \\
Donor age (year) & $30.8 \pm 11.5$ & $32.6 \pm 11.4$ & 0.024 \\
ABO-I LDLT (n, \%) & $15(11.0)$ & $22(21.8)$ & 0.098 \\
Pretransplant hepatectomy (n, \%) & $11(8.1)$ & $3(3.0)$ & 0.818 \\
Pretransplant LRT (n, \%) & $24(17.6)$ & $19(18.8)$ & \\
\hline
\end{tabular}

Continuous variables are reported as means and standard deviations. MS, microscopy; SL, surgical loupe; HCC, hepatocellular carcinoma; CTP, Child - Turcotte - Pugh; MELD, Mean Model for End-stage liver disease; ABO-I LDLT, ABO incompatible living donor liver transplantation; LT, liver transplantation; LRT, locoregional therapy including transarterial chemoembolization 
Table 2. Comparison of perioperative outcomes between two groups

\begin{tabular}{lccc}
\hline \multicolumn{1}{c}{ Variable } & MS group (n=136) & SL group (n=101) & $p$-value \\
\hline GRWR (\%) & $1.13 \pm 0.24$ & $1.10 \pm 0.22$ & 0.435 \\
Fatty change of graft (\%) & $2.2 \pm 5.5$ & $1.8 \pm 4.5$ & 0.547 \\
Graft type:right/left lobe & $129 / 7$ & $98 / 3$ & 0.410 \\
PVT (n, \%) & $32(23.5)$ & $28(27.7)$ & 0.463 \\
PVS (n, \%) & $4(2.9)$ & $4(4.0)$ & 0.667 \\
Significant hilar fibrosis (n, \%) & $25(18.4)$ & $12(11.9)$ & 0.173 \\
HA size (mm) & $2.07 \pm 0.11$ & $2.06 \pm 0.10$ & 0.576 \\
Intraoperative HA revision (n, \%) & $8(5.9)$ & $5(5.0)$ & 0.755 \\
HA reconstruction time (minutes) & $24.2 \pm 4.3$ & $20.9 \pm 6.9$ & 0.001 \\
Length of hospital stay (days) & $26.1 \pm 10.6$ & $26.9 \pm 9.4$ & 0.476 \\
In-hospital mortality (n, \%) & $5(3.7)$ & $2(2.0)$ & 0.446 \\
\hline
\end{tabular}

MS, microscopy; SL, surgical loupe; GRWR, graft-to-recipient body weight ratio; PVT, portal vein thrombosis; PVS, portal vein stenosis; Significant hilar fibrosis, fibrosis which cause difficult dissection at hilum; HA, hepatic artery

Table 3. Comparison of postoperative complications between two groups

\begin{tabular}{lcrc}
\hline \multicolumn{1}{c}{ Variable } & MS group (n=136) & SL group (n=101) & $p$-value \\
\hline Postoperative bleeding (n, \%) & $28(20.6)$ & $15(14.9)$ & 0.257 \\
Portal vein stenosis or thrombosis (n, \%) & $6(4.4)$ & $4(4.0)$ & 0.864 \\
Hepatic artery thrombosis (n, \%) & $2(1.5)$ & $2(2.0)$ & 0.763 \\
Biliary stricture (n, \%) & $6(4.4)$ & $10(9.9)$ & 0.096 \\
Biliary leak (n, \%) & $7(5.1)$ & $8(7.9)$ & 0.386 \\
RHV stenosis (n, \%) & $1(0.7)$ & $1(1.0)$ & 0.836 \\
Postoperative MHV stent insertion (n, \%) & $1(0.7)$ & $1(1.0)$ & 0.720 \\
\hline
\end{tabular}

MS, microscopy; SL, surgical loupe; RHV, right hepatic vein; MHV, middle hepatic vein

ple graft HA, small-caliber HA and ABO incompatible LDLT. Moreover, a small-caliber HA was only associated with intraoperative HA revision (OR, 0.051; 95\% CI, 0.008$0.322 ; p=0.002)$ but was also not related the use of surgical loupe (OR, 0.831; 95\% CI, 0.224-3.086; $p=0.782$ ) when adjusted for the same factors.

\section{Postoperative complications and survival}

Table 3 shows details of the main postoperative complications; no significant difference was found between the groups. There were four cases $(1.7 \%)$ of HA thrombosis: two cases in group I and two cases in group II. All thromboses were detected within 2 days after LDLT, and inappropriate manipulation for intimal detachment due to poor arterial conditions was regarded as the main cause. All thromboses were resolved with prompt re-exploration and HA revision. The resistive index and peak velocity of the HA on serial postoperative DUS were not different between the groups. Moreover, mild HA stenosis (defined as anastomotic narrowing of less than $50 \%$ with no clin- ical sign $)^{10}$ was diagnosed in 27 patients $(11.2 \%)$ by dynamic CT scan on postoperative day 14 but did not differ between the groups. No significant differences in biliary stricture were observed between the two groups. The overall patient and graft survival also did not differ significantly between the groups by Kaplan-Meier log-rank analysis $(p>0.05)$.

\section{Subgroup analysis of patients}

We divided group II into subgroup era I (the first 50 cases) and subgroup era II according to evaluate the effect of learning curve phenomenon on LDLT outcomes. According to subgroup analysis, HA reconstruction time in era I have significantly higher than that in era II $(25.8 \pm 8.3$ vs. $18.1 \pm 4.4$ minutes, $p=0.031$ ) and the increased HA occlusion rates including HAT and HA revision in era I than that in era II were shown (12.0\% vs. $3.9 \%)$ although there was no significance. As compared with microscopy group, these rates related to HA occlusion in subgroup era I have slightly higher than that in microscopy group $(5.9 \%$ vs. 
$12.0 \%, p=0.161)$.

\section{DISCUSSION}

The surgical procedures for both the donor and the recipient during LDLT are more complicated than those during DDLT. Although the operating techniques have been refined, HA reconstruction for LDLT is still considered challenging because of the risk of HAT because of the smaller arterial size, which could have dismal outcomes. ${ }^{3,6}$ In fact, the early experience with LDLT was gloomy because the rate of arterial thrombosis was as high as $28 \%{ }^{6,12}$ To resolve this problem, Kyoto's group introduced a microsurgical technique for HA anastomosis in the 1990s. ${ }^{12}$ They reported that the HAT rate was reduced from $28.6 \%$ to $5.4 \%$ with an operating microscope compared with surgical loupe. ${ }^{6,8,12}$ Subsequently, the routine use of microsurgery for HA anastomosis with higher success rates was reported in Hong Kong, Taiwan, and South Korea. ${ }^{12}$ However, compared with microsurgery on the head, neck, and extremities, there are several technical difficulties with HA reconstruction during LDLT. First, the recipient artery is located deep in the peritoneal cavity. ${ }^{1,9,13}$ The vessel ends can be more than $15 \mathrm{~cm}$ from the abdominal wall. Second, the patient's respiratory movement makes the microsurgical HA anastomosis more difficult because this movement is amplified under a microscope. Third, the recipient or graft HA often has an insufficient length to reverse a micro-clamp. Therefore, microsurgeons need time to learn the related skills and gain experience to achieve good outcomes when performing HA anastomosis., ${ }^{1,4}$

By contrast, the use of a surgical loupe is simpler than microscopy and easier to teach to trainees. ${ }^{1,9}$ As experience in LDLT grew and high-power loupe optics (4.5-6×) became available, some surgeons became comfortable using microsurgical techniques without a microscope and recent reports demonstrated that the use of surgical loupes gave outcomes for the anastomosis that were at least similar and that the procedure with a surgical loupe was superior to microscopy in terms of anastomosis time. ${ }^{1,10}$ At our institution, the postoperative outcomes were similar in the two groups although the HA reconstruction was performed by transplant surgeon with little experience in HA reconstruction during LDLT in the second period. In addition to the technical aspect, various factors such as pre- vious therapy, portal vein thrombosis, or fibrosis at the hilar area could affect the postoperative outcomes, including arterial complications, but the incidence of these factors did not differ between the groups. ${ }^{1,59}$ Moreover, multivariable regression modeling to adjust for these baseline clinical differences showed that the use with surgical loupe was not associated with an increased risk of HAT or intraoperative HA revision.

Nevertheless, many transplant surgeons still prefer to perform the anastomosis under a microscope rather than with a loupe in LDLT. The main reasons for this are the poorer vessel quality and atherosclerosis due to altered lipid metabolism and compromised liver function. They believe that the identification of healthy endothelium and management of the intimal separation under a microscope are the keys to a successful anastomosis. ${ }^{14}$ However, in the current study, intraoperative HA revision and the HAT in early postoperative period did not occur more frequently when using a surgical loupe than when using a microscope although most of these complications were caused by the poor quality of the recipient HA and subsequent intimal detachment.

However, this study had some limitations. HA reconstruction under the surgical loupe has already been performed at many transplant centers during LDLT, and many studies have demonstrated similar outcomes to those under surgical microscopy. Procedures using the surgical loupe in most studies have been undertaken by transplant surgeons with sufficient prior experience in microsurgery. On the other hand, we investigated outcomes, especially focusing on the beginner's point of view toward HA reconstruction during LDLT, and we suggest that even transplant surgeons with little experience in arterial anastomosis during LDLT could obtain good outcomes. In general, microsurgical training has been known as essential in successful arterial reconstruction. ${ }^{4,6}$ However, we suggest that it could be possible to perform HA reconstruction with surgical loupe if transplant surgeons have the basic concepts for arterial anastomosis and enough experiences in LDLT and major hepatobiliary surgery even without any real experience of microsurgical training. In fact, this surgeon has performed vascular reconstructions during LDLT except HA and enough experience in major hepatobiliary surgery.

Of course, arterial anastomosis using a surgical loupe 
could be difficult to perform in cases in which the donor HA has too small a diameter (less than $1.5 \mathrm{~mm}$ ), a short stump such as the right posterior sector or double-donor HA stumps with a smaller caliber and short length despite the right lobe grafts. We tried to apply HA anastomosis with a high-power loupe during LDLT using the right posterior sector graft and right lobe graft with double HA stumps in the early period, but immediate revision using a microscope was inevitable due to the small diameter and short stump. Therefore, we suggest reconstruction using a microscope in the cases mentioned above rather than using a surgical loupe.

In conclusion, HA reconstruction under a surgical loupe can be performed by less experienced transplant surgeons with low complication rates and yields results similar to that using an operative microscope if they have basic concepts for vascular anastomosis and enough experiences in LDLT and major hepatobiliary surgery. However, further studies are needed to apply this technique using a surgical loupe to LDLT with partial liver grafts, which have small and short HAs.

\section{ACKNOWLEDGEMENTS}

This work was supported by the grant of Research Institute of Medical Science, Catholic University of Daegu (2015).

\section{REFERENCES}

1. Tzeng YS, Hsieh CB, Chen SG. Continuous versus interrupted suture for hepatic artery reconstruction using a loupe in living-donor liver transplantation. Ann Transplant 2011;16:12-15.

2. Kim SJ, Yoon YC, Park JH, Oh DY, Yoo YK, Kim DG. Hepatic artery reconstruction and successful management of its complications in living donor liver transplantation using a right lobe. Clin
Transplant 2011;25:929-938.

3. Li PC, Jeng LB, Yang HR, Lee CC, Poon KS, Chen TH, et al. Hepatic artery reconstruction in living donor liver transplantation: running suture under surgical loupes by cardiovascular surgeons in 180 recipients. Transplant Proc 2012;44:448-450.

4. Uchiyama H, Hashimoto K, Hiroshige S, Harada N, Soejima Y, Nishizaki $\mathrm{T}$, et al. Hepatic artery reconstruction in living-donor liver transplantation: a review of its techniques and complications. Surgery 2002;131(1 Suppl):S200-S204.

5. Lee KW, Lee S, Huh J, Cho CW, Lee N, Kim HS, et al. Outcome of living donor liver transplantation using right liver allografts with multiple arterial supply. Liver Transpl 2016;22:16491655.

6. Tanaka K, Uemoto S, Tokunaga Y, Fujita S, Sano K, Nishizawa $\mathrm{T}$, et al. Surgical techniques and innovations in living related liver transplantation. Ann Surg 1993;217:82-91.

7. Inomoto T, Nishizawa F, Sasaki H, Terajima H, Shirakata Y, Miyamoto S, et al. Experiences of 120 microsurgical reconstructions of hepatic artery in living related liver transplantation. Surgery 1996;119:20-26.

8. Mori K, Nagata I, Yamagata S, Sasaki H, Nishizawa F, Takada $\mathrm{Y}$, et al. The introduction of microvascular surgery to hepatic artery reconstruction in living-donor liver transplantation--its surgical advantages compared with conventional procedures. Transplantation 1992;54:263-268.

9. Guarrera JV, Sinha P, Lobritto SJ, Brown RS Jr, Kinkhabwala M, Emond JC. Microvascular hepatic artery anastomosis in pediatric segmental liver transplantation: microscope vs loupe. Transpl Int 2004;17:585-588.

10. Marubashi S, Kobayashi S, Wada H, Kawamoto K, Eguchi H, Doki $\mathrm{Y}$, et al. Hepatic artery reconstruction in living donor liver transplantation: risk factor analysis of complication and a role of MDCT scan for detecting anastomotic stricture. World J Surg 2013;37:2671-2677.

11. Song S, Kwon CH, Moon HH, Lee S, Kim JM, Joh JW, et al. Single-center experience of consecutive 522 cases of hepatic artery anastomosis in living-donor liver transplantation. Transplant Proc 2015;47:1905-1911.

12. Kalayoglu M, Stratta RJ, Sollinger HW, Hoffmann RM, D'Alessandro AM, Pirsch JD, et al. Liver transplantation in infants and children. J Pediatr Surg 1989;24:70-76.

13. Okochi M, Ueda K, Hirose T, Okochi H, Watanabe H, Suzuki $\mathrm{Y}$, et al. A modified technique for hepatic artery reconstruction in living donor liver transplantation. Microsurgery 2010;30:541544.

14. Ulusal BG, Cheng MH, Ulusal AE, Lee WC, Wei FC. Collaboration with microsurgery prevents arterial complications and provides superior success in partial liver transplantation. Microsurgery 2006;26:490-497. 